http://dx.doi.org/10.12795/PH.1992.v07.i01.15

\title{
RETRATOS DE MUJERES: JEAN LORRAIN
}

\author{
Dolores Bermúdez Medina \\ Carmen Camero Pérez.
}

\begin{abstract}
Published on L'Echo de Paris from I 890 onwards, and afterwards on the single volume Une Fenme par jour, these 117 portraits of the artistic Paris, represent, together with his tales, the most actual legacy of Lorrain's works. Thus the writer becomes an ironical and even cruel reporter of the life in Paris at the end of the nineteenth century with one main topic: woman, a privileged character of his own universe.
\end{abstract}

Publicados a partir de 1890 y hasta 1895 en L'Echo de Paris, estos retratos del París mundano y artístico de la época representan, junto con los cuentos, el legado más actual de la obra de Jean Lorrain. Sobre la modernidad de estas instantáneas insiste Paul Morand en 1932, con motivo de la presentación de algunos de estos retratos en el volumen titulado Femmes de 1900:

«Ce qui dans son oeuvre allait durerce n'(est) pas cette poésie loïefulleresque à laquelle il déplorait tant de ne pouvoir consacrer sa vie, mais bien ses chroniques bâclées, ses propos de boulevard, ses mots portés comme des épingles de cravate, ses duels, ses batailles à coup d'épingles à cheveux avec les cocottes, toute cette pussière de Paris à quoi les critiques de l'époque lui reprochaient de gaspiller son talent»'.

Pierre Kyria en su análisis de la obra de Jean Lorrain también insiste en la importancia de este período público del escritor que se convierte en «le courriériste ironique et même cruel de l'actualité artistique, littéraire et mondaine de la capitale»². En efecto, es fun- 
damentalmente este aspecto mediático de Lorrain el que la crítica retiene hoy y el éxito alcanzado en la época de los Pall-Mall se ve reforzado en la actualidad por la publicación de relatos breves aparecidos en periódicos, tales como Histories de masques o esta Femme parjour, que aquí nos ocupa, asícomo otros textos (Très Russe, Les Noronsoff, etc.), dejando pues de lado sus largas novelas como M. de Phocas, M. de Bougrelon o La maison Philibert, por no hablar ni de su poesía ni de su teatro que parecen estar hoy definitivamente muertos.

En la selección y revisión realizadas por Pierre-León Gauthier para la publicación de Femmes de 1900, amalgama de Une femme par jour y de Vingt femmes, estos retratos femeninos se encuentran enmarcados por la figura de Salomé, el primero data del 30 de noviembre de 1891 y el último del 5 de noviembre de 1893 (ambos publicados en $L$ 'Echo de Paris). Aunque estos retratos no figuran en Une femme par jour, nos serviremos de ellos para presentar el esbozo inicial de la representación de la mujer según Jean Lorrain, que se muestra en esto deudor de su época y de sus maestros más admirados: Gustave Moreau y J.-K. Huysmans. Lujuriosa, histérica, mujer-verdugo del hombre, Salomé quintaesencia el retrato-tipo de la mujer-género, es, en términos de Huysmans: «La bête mostrueuse, indifférente, irresponsable, insensible, empoisonnante de méme que l'Hélène antique, tout ce qui l'approche, tout ce qui la voit, tout ce qu'elle touche» ${ }^{3}$. Pero no por ello Lorrain la rechaza, ya que como bien señala Paul Morand «qui est donc aussi femme que cet homme lorsqu'il parle des femmes? $\gg^{4}$. Esta identificación primera se hace no obstante mirada distanciada y crítica cuando el autor nos presenta esas «femmes d'été», que caracteriza, sobre un fondo saloméico, a partir de algunos rasgos que combinados de forma diversa, ultiman el retrato de cada tipo de mujer, variante concreta y parisina de esta primera eva-salomé.

Si analizamos los títulos de estos retratos, encontramos en Lorrain un aparato titular a dos niveles, de los cuales, el primero está constituido por el título general: Une fenme par jour, y el segundo por la lista de títulos particulares: «La pucelle de Belleville», Les amoureuses de Cléopâtre», «Phrynette», «La Cocosotte», «La dame du jour de l' an», «Madame Coqenpot», «Les Grimpettes», L'Etrangère», «LaProvidentielle», «L'Araignée du cimetière», «Fleur de fortifes», "Celle qui s'cn va»... Estos títulos, pertenecientes a lo que Genette denomina paratexto ${ }^{5}$, cumplen tres funciones principales que, siguiendo a los titulólogos ${ }^{6}$, designamos con los términos de identificación, designación y puesta en relieve, refiriéndose el primero y el tercero al conjunto de la obra, y el segundo a su contenido.

El libro ha conservado el título general de origen, Une femme par jour, título de la serie periodística que Lorrain decide firmar con otro nombre, el de Restif de la Bretonne, reemplazado más tarde por el de Raitif de la Bretonne debido a las protestas de un

J.-K. HuYsmans, A Rebours.

Paul. Morani), op. cit., p. VII.

G. Gent:TT1:, Seuils (Paris, Seuil, 1981), p. 9.

Cr. Grivf, Production de l' intérêtromanesque (Mouton, La Haye, 1973), pp. 166-181, y L. How: Lamarque du titre (Mouton, La Haye, 1982), p. 17. 
descendiente del escritor. Lorrain atribuye así falaciosamente sus textos a un autor desconocido, haciendo de su obra un apócrifo. Los problemas planteados por tal práctica (motivo de la elección o efecto producido en el lector por la utilización del seudónimo), no serán analizados en estas páginas, pero queremos, no obstante, señalar la importancia que, en nuestra opinión, debería concederse al efecto-seudónimo en el marco de estudio de las condiciones de recepción de las obras.

El título general, Une femme par jour, sirve en primer lugar para nombrar e identificar el libro de Lorrain sin riesgo de confusión. También designa el contenido de la obra y aparece como un título temático literal ${ }^{7}$, que anuncia directamente el tema central: la mujer, figura privilegiada del universo lorrainiano. La referencia temporal que el autor añade a este tema central encuentra su explicación lógica en el medio de difusión de los textos: el periódico.

La tercera función del título, la puesta en relieve del libro, intenta atraer al público; «función seductiva», se revela evidentemente subjetiva según los receptores. Dos puntos de interés pueden ser señalados aquí: el del público que se interesa particularmente por el universo femenino y de los receptores, que se sienten atraidos por las curiosidades de una sociedad y una época, la de finales del siglo XIX, información ésta que el título general no nos ofrece (y que no tenía por qué ofrecernos, dado que la publicación de los tex tos era contemporánea de la recepción de los mismos), pero de la que nosotros, lectores ulteriores, disponemos gracias a la instancia del prefacio.

La lista de los títulos designa el contenido de cada retrato y se reparte en distintas categorías:

1. la de nos nombres propios: «Madame Baradin», «Madame Coqenpot», «Réjane», «Yvette»...

2. la de las metáforas: «Les amoureuses de Cléopâtre», La Pompe funèbre», «Les Grimpettes»....

3. la de los predicados calficativos y funcionales: «La Cocosotte», «La Couveuse artificielle», «La Débutante», «L'Angoissée»...

4. la de las metonimias y/o metáforas: «Pietà de Mazas», «Fleur de fortifes», «Fleur de chic»...

5. la de las perífrasis: «Celle qui s'en va», «Celle dont on ne parle pas», «Celle de chez. Bodinier», «Celle qui reste»...

6. la de los circunstanciales espacio-temporales: «La Dame du jour de l'an», «La Dame du kiosque», «Dames de plage»...

En estos títulos, la presencia del deíctico tiende a establecer una situación de discurso ${ }^{8}$. Generalmente se considera que los deícticos en un texto escrito tienen una función

7 G. GintrTt, op. cit. pp. 78-85, propone el adjetivo temático para calificar los títulos que se reficren al contenido del texto y remático para los que se refieren a una calificación genérica o formal.

* Cfr. M. Cor.t.), «La dimension du déictique», Littérature (mai, 1980), pp. 62-76. 
esencialmente anafórica, ya que remiten al contex to lingüístico anterior. Sin embargo, en nuestro caso ningún contexto precedente permite identificar las alusiones contenidas en los títulos. Habría pues una especie de laguna inaugural, un vacío de significación, que el lector intenta cubrir; es muy probable que si Lorrain no explicita estas alusiones, es porque aquello de lo que habla se refiere a una sociedad y a una cultura que posee en común con su público. Este público, que oponemos a nosotros, lectores, se inserta en la configuración discursiva del título y asume el papel de locutor. Si para nosotros, lectores del siglo XX, no hay referencia precisa, para el público de Lorrain y de L'Echo de Paris, esta referencia existe y una complicidad se establece entre narrador y narratorio por el hecho de compartir la misma situación socio-cultural. La mujer inicialmente ausente deignada por el deíctico, se hace presente gracias al pacto existente entre los locutores. Pensamos que si Lorrain se dirige a un locutor, como si se tratase de una verdadera situación de discurso, es evidentemente a los lectores de $1890-95$ y no a nosotros. En efecto, es el público de Lorrain y de L'Echo de Paris el que será capaz. de encontrar inmediatamente el referente y asignar al retrato un nombre propio. Esta operación será cada vez actualizada en el presente de la lectura de tal manera que también nosotros, lectores del siglo XX, intentaremos cubrir el vacío de este tipo de títulos, si bien la búsqueda del referente será sin duda mucho más difícil.

En Une femme par jour ${ }^{\text {, }}$, Jean Lorrain nos ofrece 117 retratos de mujeres, de los que, por razones de economía, sólo hemos retenido los más significativos. Proponemos hacer, en el interior de este conjunto, una clasificación, que revela la existencia de dos grandes grupos:

1. El de las mujeres que representan el París artístico, literario y mundano de la época y cuyos relatos pertenecen al campo de la nominación.

2. Aquél otro, mucho más restringido, de las mujeres que representarían el ideal femenino del autor, y cuyos relatos pertenecen al campo de lo que llamamos, siguiendo a Vouilloux ${ }^{10}$, la narración.

El primer grupo admite a su vez una nueva clasificación, que nos permite diferenciar las artistas (de teatro o de variedades) de las literatas («militantes» o víctimas) y de las mundanas (burguesas, aventureras, prostitutas o perversas).

El primer retrato del grupo «mujeres artistas de teatro» se litula «La pucelle de Belleville» y lleva como epígrafe un texto de Michelet:

«Née sous les murs mêmes de l'église, bercée au son des cloches et nourric de légendes elle (Jeanne d'Arc) fut une légende elle-même rapide et pure de la naissarce à la mort»».

" Utilizamos la edición de M. Di:sbruìres (Paris, Christian Pirot, 1988). Los números de páginas que a partir de ahora indicaremos se refieren a esta edición.

10 B. Vout.1 oux, «La Venelle du sourd. Sur la référence et l’allusion: Gracq, Cezanne, Goya», Poétique, 62 (avril. 1985). 
Este epígrafe cumple su función canónica de comentario de texto, subrayando su significación indirectamente: la mujer pintada por el autor se asemeja a la figura legendaria de Juana de Arco por sus cualidades y sus funciones. Los predicados calificativos están ya contenidos en el mismo epígrafe: esta mujer fue una «légende rapide», los funcionales, por el contrario, no quedan claros más que con la lectura del texto, cuando nos enteramos de que dicha mujer ha representado en el teatro de La Halle el papel de Juana de Areo.

Este epígrafe, por el nombre de su autor (Michelet), tiende también a esclarecer o más bien a justificar el título de la crónica; título que está constituido por un préstamo parcial, no del tex to citado, sino de las referencias de éste, concretamente el título del capítulo III, del libro II de la Jeanne d'Are de Michelet: «La pucelle d'Orléans»; artículo y nombre han sido mantenidos, mientras que la referencia local ha sido transformada.

«La pucelle de Belleville» aparece como la reseña de la representación de Jeanne d'Are por Mme Eugénie Segond-Weber, actriz dramática (1867-1945). La mujer cuestión de esta crónica es nombrada al principio del texto, nominación que permite pasar fácilmente de la referencia escrita al referente real: Mme Segon-Weber, mujer escrita, es también Mme Segond-Weber mujer real, que debutó en La Comédie Française en 1887. Actuó luego, durante once años, en el Odéon, volviendo a La Comédie Française en 1990 para representar a las heroinas de la tragedia clásica.

El retrato de esta mujer se realiza desde dos puntos de vista, diferentes y complementarios:

1. el del escritor-periodista

2. el del público, verdadero narratario de esta crónica.

El discurso descriptivo del escritor se basa principalmente en el contraste y el juicio de valor. La visión de Mme Segond-Weber representando a Juana de Arco en el teatro de La Halle establece, en el espíritu de Lorrain, un contraste con su bien amada Sarah Bernhardt representando el mismo papel en La Porte Saint- Martin. A la figura de Juana de Arco, Sarah «avait prêté le charme inoubliable de ses gestes et de sa sihouette pure» (p. 253). Mme Segond-Weber, sin embargo, «aurait bien dû s'en tenir à sa petite héroine highlander et patriotique (...) du beau drame de M. Coppée: Les Jacobites» (p. 253), pues como señala el Lorrain moralista y juez: «on ne touche impunément aux légendes».

Mediante el contraste Lorrain subraya el valor significativo de la descripción" ${ }^{\prime}$, descricpión que quiere ser realista y pone en escena a un público, aquél al que el escritorperiodista se dirige, que garantiza la veracidad de las descripciones inscribiéndolas en un contexto y una realidad existentes. A nivel de la escritura, el empleo de los demostrativos tiende a probar el referente: «Cette démarche lourde, ces gestes brusques, ces façons presque

1 Cfr. P. Inвt-kт, «Sémiostyle: la description chez Balzac, Flaubert et Zola», Littérature (mai, 1980)), pp. 106128. 
de commère, de se planter et de se camper, mais cela serait sifflé au théâtre des Batignolles» (p. 254). Por otra parte, las llamadas al público mediante la utilización del pronombre «vous» («vous voyez d' ici les clichés indiqués de la touchante rencontre entre la fille du prolétaire et le sensible auteur des Humbles», (p. 254), permite instaurar una situación de discurso y de comunicación entre el narrador y sus narratarios. No creemos necesario insistir en el hecho de que tanto el demostrativo como el pronombre funcionan claramente como signos evidentes de la escritura realista.

El realismo y la veracidad de la crónica, Lorrain intentará acentuarlos con la introducción de lo que hemos denominado el punto de vista del público, que viene a expresarse en el texto mediante el empleo de un discurso indirecto libre, que el contex to nos permite atribuir no al escritor, sino a los receptores, tomados aquí como testigos del acontecimiento:

«Ce fut une révélatin au soir de la primière. Où diable Paul avait-il découvert ce profil tragique, ce fier sourire aux lèvres sinueuses? Et le nom de Rachel courut dans la salle». (p. 253).

Al referir el discurso del público, Lorrain adquiere la condición de fiador de la autenticidad de los hechos.

Siguiendo en este mismo campo de las actrices de teatro, con «Les Amoureueses de Cléopâtre», pasamos del retrato particular a un retrato general (anunciado ya por el título en plural) válido para toda una clase de mujeres, que Lorrain sigue describiendo en relación con la mujer actriz, la verdadera diosa, la reina de la escena: Sarah Bernhardt, la emperatriz Cleopatra.

El objeto de descripción (de pintura) se anuncia ya en el título, que instaura así, como bien señala Philippe Hamon ${ }^{12}$, un «système d'attente destiné à la lisibilité de la description qui suivra».

El tema introductor, que por su presencia al principio y al final del texto confiere a éste una estructura circular, y el paradigma del léxico que lo asume, pueden ser fácilmente identificables, dado que la serie elegida para el retrato de estas mujeres es una serie absolutamente previsible, que revela la utilización de un léxico fundamentalmente prosopográfico, mediante el que se describe a estas mujeres por su aspecto, sus trajes, sus cabellos, sus ojos:

«(...) toutes reconnaissables à leur allure haride et garçonnière, le feutre mou crânement posé sur leur cheveux roussis au henné et frisés à la Titus, la jaquette de tailleur ouverte sur le gilet de drap olive, toutes d'une pâleur de morte sous leur coiffure androgyne et les yeux cruels et fixes entre leurs cils mouillés de fard». (p. 136).

Los predicados privilegian de manera evidente una serie de términos estereotipados para pintar un tipo de figura femenina de caracteres masculinos, que determinados 
decadentes, Joséphin Péladan por ejemplo, se complacerán en describir convirtiéndola en protagonista de sus obras.

A partir de Iso calificativos «hardie» y «garçonnière», pasando por la «jaquette de tailleur ouverte sur le gilet», llegamos al predicado capital: «androgyne», con el que Lorrain muestra explícitamente el tipo de mujer que quiere describir, mujer «bien fin de siècle», como afirma el mismo autor (p. 136), el andrógino será también «le sexe artistique par excellence», representado en las criaturas de Leonardo da Vinci:

«Léonard a trouvé le canon de Polyclète, qui s' appelle l'androgyne... L'androgyne este le sexe artistique par excellence, il confond les deux principes, le féminin et le masculin, et les équilibre l'un par l'autre. Toute figure exclusivement masculine manque de grâce, toute autre figure exclusivement féminine manque de force, Dans la Joconde, l'autorité cérébrale de l'homme de génie se confond avec la volupté de la gentille femme, c'est de l'androgynisme moral» ${ }^{13}$.

Este androginismo, que Lorrain pinta en sus retratos periodísticos, constituye uno de los temas fundamentales de su obra literaria. En Monsieurde Phocas, una bailarina griega es calificada de «Vénus Alcibiadée», y Sonia, la protagonista de Très Russe, explica a Mauriat que no se entrega a él porque «la chasteté est l'extrême désir».

Estas mujeres son espíritus diabólicos que alían la inocencia y el vicio, como lo muestra bien el retrato de Phrinette, joven artista de variedades, perversa y fatal:

«... Phrynette et ses quinze ans, ses yeux velus et noirs, ses tombantes paupières, Phrynette et sa peau claire, sa maigreur provocante, ses déhanchements canailles et la saveur verte de sa bouche de vierge, de son corps élancé! (...) Phrynette, animale et grisante petite femelle dont la chair attire et tisonne les adolescents bruns et leur fait mordre en rêve leur oreiller inerte, Phrynette pour l'amour de laquelle coulent les larmes des mères et s'entrebaîllent les coffres-fort des vieux qu' elle fait saliver (...) sa grâce inconsciente de jeune bête en folie, malfaisante et perverse (...). C'est qu'elle sent à plein nez l'atelier et le vice» (pp. 44-45).

Si en «Phrynette» $y$ «Les Amoureuses de Cléopâtre» podemos hablar de referencia literaria, la crónica de «La Débutante», primer retrato del grupo que hemos llamado de las «literatras», constituye en sí mismo una pura intertextualidad literaria. Lorrain nos presenta esta vez el retrato de una mujer anónima, ya que es representativa de toda una clase, la de las imitadoras desafortunadas de la moda literaria. La comparación y el contraste que de clla resulta se establece entre esta clase de mujeres y Rachilde, modelo que pretenden imitar.

13 "Epilogue», en Leonardo da Vinci, Conférences Florentines (Milan, Trèves, 1910), p. 318, citado por M. PRA\%, La chair, la mort et le diable (Paris, Denoël, 1977), p. 277. 
El tex to comienza por un primer párrafo fulgurante de prosopografía y etopeya, donde los rasgos físicos («grande taille longue et souple», «chevelure blonde») van acompañados por caracteres morales que, en su contexto, denuncian ingenuidad y vicio: «avec ses ycux limpides, aux cils emperlés d'eau, candides, des yeux de fleur ou d'enfant; elle aussi (...) ingénument presque, est descendue dans cette sentine, et de ses mains fines et délicates a remué cette houe» (p. 190)). Tras este comienzo, el tex to desaparece para dar paso al intertexto: el marqués de Sade, Leshos, Rachilde y su Monsieur Vénus, Barbey D' Aurevilly, Catulle Méndès. Estos nombres propios encuentran su referente en el mundo real y son identificados y localizados por el lector, lo que permite a Lorrain economizar en la descripción.

El hecho de nombrar a estas personas, como muy acertadamente ha señalado B. Voilloux, «en redant prévisibles les principaux traits distinctifs du personnage référentiel, limite considérablement les expectatives du lecteur et, libérant en celui-ci les associations et les connotations ayant le nom pour noyau, lui fait accomplir une part du travail dont le produit n'est autre que la représentation du tableau» ${ }^{14}$.

Este cuadro es el que nos ofrece la literatura decadente que se complace en proclamar el ideal del andrógino, del amor asexuado y del sadismo. En efecto Rachilde proclamaba en $1884 \mathrm{el} \mathrm{ideal} \mathrm{del} \mathrm{andrógino} \mathrm{en} \mathrm{su} \mathrm{Monsieur} \mathrm{Vénus,} \mathrm{libro} \mathrm{por} \mathrm{el} \mathrm{que} \mathrm{sintieron} \mathrm{gran}$ entusiasmo Barbey d' Aurevilly y Maurice Barrès ${ }^{15}$. Jean Lorrain, por su parte, hará un retrato de su autor: «Mademoiselle Salamandre», publicado en Le Courrier Français del 12 de diciembre de 1886 :

Une pensionnaire d'allures sobres et réservées, très pâle, il est vrai d’une pâleur de pensionnaire studieuse... une vraic jeune fille un peu mince, un peu frêle, aux mains inquiétantes de petitesse, au profil grave d'éphèbe grec ou de jeune Français amoureux... et des yeux-omh! les yeux! des yeux Iongs, longs, alourdis de cils invraisemblables et d'une clarté d'eau, des yeux qui ignorent tout».

¿Cómo no ver en este retrato de Le Courrier Français muchos de los rasgos presentes en el de L'Echo de Paris?

La intertextualidad literaria de este retrato será para Lorrain el medio más favorable para probar lo bien fundado de su juicio de valor. Al citar a B. d'Aurevily, C. Mendès y la misma Rachilde, Lorrain muestra la clave del éxito de esta literata cerebral: «elle avait inventé la chaste prostitution» (p. 191). Y junto a este monstruo literario, la corte de las imitadoras contra las que Lorrain dirige su locuacidad feroz:

«Mais qu'espèrent aujourd'hui du public et même de la presse ces sousmaîtresses de l'étrange, toutes ces pseudo-Rachilde (...) ô Baudelaire mal

14 B. Voun. oux, «La description du tableau dans les Salons de Diderot», Poétıque. 73 (février, 1988), p. 41.

15 M. Barki:s escribió un Prólogo para la edición de 1889. Define en él el libro como «spectacle d'une rare perversitén. 
digéré, d’Aurevilly mal compris, Verlaine aigrissant et surissant conme un vin trop nouveau, dans le vide enfantin d'une pauvre jolie tête!» (pp. 191192).

Mordaz e irónico se presenta también el retrato de Philaminte, objeto de descripción en «La Couveuse artificielle», título metafórico por el que Lorrain designa el tipo mismo de la literata militante y mundana, señora de salón ridícula y mediocre. El retrato es cruel e irónico, combinando prosopografia y etopeya. Lorrain se sirve de un discurso estilizado al referir las palabras de esta mujer, que el autor imita empleando una escritura fonética con vistas a reproducir el habla del personaje:

«(...) elle s'ètablissait, la bonne dame, de trouver que rien n'était plus littéraire que ses invités et son salon» (p. 236).

La ironía impide considerar este discurso como un signo de consonancia entre escritor y personaje, obligándonos pues a interpretarlo como un signo de disonancia manifiesta para subrayar el ridículo de esta «couveuse artificielle».

Muy distinta será la actitud de Lorrain para con esas otras mujeres, literatas también, pero un tanto chifladas y debilitadas por la vida literaria y mundana, que hemos agrupado bajo la denominación de «literatas víctimas». Para ilustrar este nuevo tipo de figura femenina citaremos la crónica titulada «La fille aux mains coup...ables». Más que un retrato, este tex to constituye la defensa de una asesina, una mujer un poco loca que, bajo la influencia de la literatura perversa, satánica y sádica de la época, llega a matar a su amante. Como las scudo-Rachilde de «La Débutante», esta mujer también ha dirigido mal las obras del momento (Les Veilleuses, La fille aux mains coupées, Madame la Mort), pero la ironía feroz aque atacaba a las primeras se transforma aquí en piedad y compasión, y la disonancia que separaba antes autor y personaje se convierte ahora en complicidad y consonancia. A nivel discursivo, el discurso irónico desaparece para dar paso a un discurso indirecto libre muy adecuado para la expresión de esta consonancia, yá que lunde, como es sabido, la voz del narrador y la del personaje:

«...le savait-elle, seulement, elle-même pour le seul plaisir peut-être d'enfoncer de l'acier dans cette chair grasse et rose et de voir le sang rouge, en un jet, en giclet!» (p. 308).

La estructura del texto también cambia; la crónica no termina recobrando el objeto descrito, como sucede en la mayoría de los retratos, sino con un grito de absolución, que perdona a la culpable, como si del veredicto de un jurado se tratase: «Et mes mains t'ont obsoutc, pauvre cerveau fêléc!» (p. 310).

Una vez eshozado este primer grupo de mujeres del París artístico y literario, pasamos -siempre en el terreno de la nominación- al análisis del segundo grupo de retratos lemeninos, que abordaremos, aunque las técnicas sean básicamente las mismas, desde una perspectiva algo diferente, es decir, de manera más global. En efecto, dentro de este grupo de mujeres «mundanas» del que vamos a ocuparnos inmediatamente, se integraría un cierto número de retratos cuyos rasgos comunes, es decir, sus predicados de base, son hastante limitados: sexo y dinero. A estos habría que añadir, en las representaciones 
particularizantes, la ambición, en el caso de las mujeres aventureras, la mediocridad en las burguesas y el sadismo en las que llamaremos «perversas»; sexo y dinero consiluyen, en cualquier caso, los predicados de las prostitutas.

Por lo que se refiere a la organización, estos textos responden, como los anteriores, al esquema establecido por Philippe Hamon para la descripción: Personaje + Forma + Tema (la mujer) + Nomenclatura, dividida en dos grandes subgrupos: $\mathrm{N} 1$, las artistas que acabamos de analizar, y N2, las mundanas que veremos inmediatamente, subgrupo caracterizado por predicados bien calificativos o bien funcionales. En nuestro caso, evidentemente no hay relación entre narración y descripción: la descripción garantiza por sí misma, en tanto que retrato, la organización del texto. La descripción asume pues, en líneas generales, algunas de las funciones que tradicionalmente le han sido asignadas: la función focalizadora, la función organizadora y, por último, debido al «efecto de realidad» que produce, podríamos pensar también en una función decorativa.

En el desarrollo de la fórmula establecida por Hamon, habría que hacer algunas precisiones con relación a lo que él llama «personaje». En los retratos de Lorrain, puesto que no hay relato, no hay personaje propiamente dicho: el vehículo introductor de la descripción viene -la mayoría de las veces- directamente asumido por el narradorscriptor, que confiere a estos retratos bien sea un carácter de verosimilitud, bien una distancia crítica o irónica. La mirada del narrador la palabra del scriptor-puesto que asume una rúbrica en el periódico- constituiría lo que Hamon llama la forma de la descripción («mirar», «hablar de») cuya tercera manifestación -«actuar sobre»-será retomada más tarde en la descripción de las mujeres narradas. Este aspecto referencial es esencial, ya que estas crónicas están dirigidas precisamente a un público lector del periódico que, o conoce a los personajes de los que habla Lorrain, o desea adivinarlos cuando las referencias están algo veladas. Es, por lo tanto, en un contex to mediático donde deben ser analizados estos textos, que obtienen su popularidad y su éxito precisamente de la complicidad o de la no-complicidad según el alcance de las invectivas establecidas entre el cronista y sus lectores.

\section{LA PRESA Y EL CORRAL}

El carácter mundano de las mujeres de Lorrain se despliega en dos grandes escenarios: los salones y la calle, lo que, de entrada, ubica a estas mujeres en dos espacios divergentes que, con toda seguridad, implicarán la descripción de dos ambientes opuestos, uno interior, el otro exterior; siendo el gabinete el lugar de intersección espacial.

En los salones encontraremos a las burguesas, por un lado, y a las aventureras, por otro. Partiendo de un mismo predicado de base - la pasión por el dinero o, mejor, la necesidad de hacer ostentación de él- los retratos de estas mujeres de salón manifiestan dos tipos de predicados calificativos: la mediocridad para las burguesas y la ambición para las aventureras.

Planteadas las líneas generales de análisis, pasemos ahora a detallar algunas de estas decripciones. En general, las burguesas no son caracterizadas físicamente; sólo se las ve 
a través de su entorno. Por entorno entendemos tanto la situación económico-familiar (marido, hijos, rentas, medio del que proceden, vecindad...) como el marco doméstico puramente espacial en el que se supone que viven: casa, mobiliario, ajuar, etc. Así pues, en «La Cocosotte» podemos leer:

Jeune, de vingt deux à trente, mariée depuis peu et toute nouvelle encore dans son menage, la cocosotte est essentiellement parisienne: le mari, généralement employé, ingenieur ou architecte de la Ville, dans les six à huit mille d'appointement, trime comme un forçat pour subvenir au luxe illusoire de Madame, dont le père dans les affaires, courtier ou financier marron, a négligé de verser la dote et oublié régulièrement d'en payer depuis la rente annuelle» (p. 35).

Raramente descritas físicamente, contrariamente a los rasgos prosopográficos que dominan en los retratos de las artistas, o, en último extremo, caracterizadas a grandes rasgos (gorda, joven, fea, guapa...), las mujeres burguesas se distinguen también por su forma de vestir:

«La toilette, neuve de la tête aux pieds, depuis le corset de satin mauve jusqu'au fin bout des étroites botines, Madame Coqenpot tout battant neuf ce jour-là: c'est une tradition de famille. Gêlerait-il à Pâques pour les Coqenpot, c'est le printemps, et, si elle laisse aujourd'hui leurs fourrures et leurs snow-boots aux petites Coqenpot, c'est bien pour ne pas contrarier Monsieur» (p. 313).

En este marco fijo, cerrado e inmóvil, desarrollan las burguesas su actividad, que es prácticamente ninguna a no ser la de mantener el estatus que exige la clase y que se circunscribe a un cierto dinamismo en las cuestiones del vestuario, en las cuestiones familiares (educación o, lo que es lo mismo, transmisión de valores inamovibles) y en las cuestiones sociales, limitadas a relaciones de vecindad, de barrio (portera, comerciante...) y a prácticas socio-religiosas.

A partir de los rasgos que hemos esbozado hasta el momento, se adivinará fácilmente que, vistas en su conjunto, estas mujeres hacen gala de la más insoportable mediocridad:

«Epousées por leur dote ou leur influence, ce sont même laides à ternir les miroirs, les belles Mmes Parleydondemoy, dont l'unique métier est, dix mois sur douze, de s'habiller et de se deshabiller, de se maquiller et de se poudrer, de s'attifer et de se diamenter, et pomponnées, ciffées, empanachées, de se promener et de se surmener, de s'exiber et de se détailler, en robe courte le matin, et les seins nus le soir, dans tous les endroits du monde et de Paris où leur présence peut être signalée à la confiante admiration des lecteurs d'autant plus crédules qu'ils les ignorent!» (p. 159).

Mediocridad que se convierte directamente en estupidez en los casos en los que no ha habido «pasado»-sexual, se entiende-que confiera una visión más astuta y distanciada en el manejo y «conocimiento» de los hombres, es decir, en su dominio, puesto que en la reserva parisina, en este coto privado que son las relaciones hombre-mujer descritas por 
Lorrain, todo ocurre entre el «corral» y la «presa»: en la burguesía, aunque las relaciones sexuales no desaparezcan completamente, son los aspectos alimenticios los que prevalencen en la rusticidad de estas mujeres domésticas. A veces se consideran ambos aspectos:

«(...) parfois elle (la Grimpette) essaie bien de louvoyer et de pénétrer dans l'arche au bras de quelque amant titré, blasonné, signeur de noble terre, elle arrive alors au scandale, au lieu de s'en tenir au ridicule, et c'est tout ce qu'obtient ce petit gibier de premières et de salons promenoirs dont un clubman d'esprit a résumé l'utilité d'un mot: "petites volailles truffées de vanité, parfois mangeables à la sauce adulère"» (p. 161).

Esta sexualidad convertida en rito alimenticio se manifiesta igualmente en otros relativos como el de Madame Coqenpot en el que el carácter de «ave de corral» que se percibe claramente en el nombre propio está explícitamente reforzado en el texto:

«(...) rayonante au milieu des génuflexions des demoiselles de boutique, matronne enviéc des mères moins heureuses, elle fera à voix de tête flûtée et suraigûe, sa commende pour le soir: "Tourte au gibier... qu'importe... il me faut du gibier, j'ai vingt personnes à dînner... Un jambon, sauce madère... et... surtout des ris de veau", discutant le menu afin qu'on en ignore daignant consulter, en bonne petite mère, le goût de ses poussins pour le choix des gâteaux» (p. 314).

Ambos aspectos, el «corral» y la «presa», se dan cita igualmente en los salones de otro tipo de burguesas, las aventureras, subgrupo con el mismo predicado de base que los anteriores -pasion por el dinero-pero cuyos predicados calificativos se inclinan más bien hacia la ambición a través del sexo:

«Elle est surtout ćtrange, ce qui est le plus sûr moyen de demcurer étrangère partout, et toute sexagénaire qu' elle soit aujourd'hui, porte encore avec elle une odeur de péché, une saveur d'adultère, ce fumet de haute venaison, cher au palais des blasés et des friands d'amour» (p. 118).

Aunque vivan en París, la mayoría de estas mujeres son extranjeras (polacas, rusas... $)^{16}$ y en su descripción, se distinguen de las burguesas, principalmente, por una mayor atención a los rasgos físicos, indicio, en el retrato lorrainiano, de una mayor promiscuidad. Como en el caso de las burguesas también es el entorno lo que describe a Ias aventureras, sólo que en este caso es, evidentemente, mucho más lujoso:

«Avenue Henri Martin, un merveilleux hôtel du plus pur style Louis XV, aux boiserics rocaille copiées exactement sur celles de Rambouillet, panneaux de soie peinte, tels des éventails, par les Leloir te Abbéma, fenêtres à chassis et portes tout en glaces, mobilier de haut goût éxécuté après maintes et maintes visites à la Bibliothèque et au Garde-Meuble, cuivres de Gouttière et cartons de Watteau, et dans la chambre à coucher 
royale, celle de Madame de Pompadour à Choisy, les plus belles étoffes de manufactures de Lyon, gros de Tours saumon et brocard d'argent, satins aurore, iris pâle et citron, chinoiseries comme en rêvait Goncourt, statuettes d'Houdon, bombonières de Galuchat et meubles vert céladon, consoles d'un modèle introvable aujourd' hui, aux fïnes sculptures évidées et fleuries... et dans le boudoir, contre un paravent peint par Fragonard, un service de thé rose Dubarry» (p. 277).

Por lo que se refiere a sus «actividades», el rasgo más característico es la ausencia de relaciones familiares y religiosas, compensada con un dinamismo social cosmopolita que afecta tanto a terrenos más o menos artísticos como a otros más mundanos y frívolos.

Esposas morganáticas o amantes declaradas, estas extranjeras presentan un carácter de «corral» más evidente que sus riväles burguesas pero puesto que ambas son cazadoras de hombres, se aferran a ellos para vampirizarles la sangre social, es decir, el dinero.

\section{EN EI. MATADERO}

Si las mujeres del grupo anterior parecen estar representadas en función de ciertos ritos temporales («La dame du jour de l'an», «La Dame qui recevra après Pâques», «L'Enchantée du Vendredi Saint») que marcan con una cierta importancia la mediocridad de su vida cotidiana, en los retratos de las prostitutas el entorno abandona el carácter puramente indicial de los anteriores retratos para garantizar un rol funcional importante: los tres que hemos destacado -«L'Araignée du cimetière», «Celle qui fait les gares», «Fleur de fortifes»-subrayan precisamente este aspecto en su título mismo: el cementerio, la estación y el arrabal son los espacios en los que se desarrolla la actividad de estas mujeres cuyo predicado de base común es la actividad sexual. A éste puede añadirse, en el caso de las prostitutas, un predicado funcional: la búsqueda de dincro. Con un predicado funcional diferente, la húsqueda exacerbada e incluso sádica del placer tendremos la descripción del último gran subgrupo de estas mujeres «nominadas», predicado funcional sanguinario característico de cierta categoría de mujeres a las que, cautelarmente llamaremos «perversas» o «sádicas».

En ambos grupos, la descripción física de los personajes femeninos debe desempeñar forzosamente un papel importante. Así comienza, por ejemplo, «La Pompe funèbre»:

«Joris Karl Huysmans l'a burinée en trois coups d'ongle dans son étude sur Félicien Rops: face glacée et vide, canaille et dure, yeux limpides, au regard fixe, le regard fixe et cruel des Tribades, bouche un peu grande. fendue droite, nez régulier et court» (p. 106).

Por lo que se refiere al espacio propiamente dicho en el que se desarrollan estos vagabundeos femeninos, se observa en él un carácter marginal y suburbial, exterior. Las prostitutas se encuentran en la calle, mientras que a las «sádicas» podemos encontrarlas más fácilmente en tugurios de mala muerte. En este caso, la descripción se torna marco: los prodecimientos descriptivos asumen un valor que abandona el aspecto puramente 
ornamental o decorativo de los retratos burgueses, desempeñando una función organizadora del relato. Frente a las instantáneas burguesas, el retrato de las prostitutas y de las perversas es móvil o, mejor, permite la movilidad de las siluetas; el vagabundeo femenino, la búsqueda de dinero a través del sexo, tiene lugar en la calle. El movimiento hace irrupción y la acción, casi siempre, está minuciosamente espacializada:

«C'est un sourire de coin, un psitt psitt, une oeillade... Si le client avance, les fossés son tout près, la femelle y descend: si l'homme est un turban artisan de banlieu, ouvrier mégissier, briquetier ou puisatier, la gadoue s'exécute la brutale idylle se dénoue en plein air, tâtonnante dans l'onbre, à l'abril d'une porte, au revers d'un talus» (pp. 20-21).

«Turista erotómana de la Perversidad», «Vicio errante», «asidua de tugurios y de antros», «tristes almas, desde la infancia ya embarcadas para el más allá», «equívoca vagabunda qu uno se encuentra», la interacción entre el medio y el personaje se acentúa en los retratos de estas mujeres que deambulan por París: la calle es su gran focalizador. La inscripción e interacción del espacio de estas representaciones lleva inevitablemente aparejado el empleo de procedimientos metonímicos en la descripción. Efectivamente, siguiendo la clasificación establecida por Mieke Bal en su artículo «Descriptions. Etude du discours descriptif dans le texte narratif» estaríamos ante descripciones referencioretóricas cuyos «éléments sont combinés selon la contigüité et selon leur fonction thématique» ${ }^{17}$.

Probablemente es también su dependencia del entorno, su imbricación, lo que constituye la particularización de estos retratos.

En cuanto a los títulos, constatamos una tendencia hacia la metaforización: «La Pompe funèbre», «L'Araignée de cimetière», «Fleur de fortife»... como si, por parte del escritor, hubiera una especie de mirada transformadora, una voluntad de metamorfosear, de «literaturizar» si se quiere, el retrato de estas mujeres, perdiendo quizá la distancia irónica de los retratos burgueses, pero sin disminuir por ello el nivel de crudeza y de violencia que los ha inspirado. A fin de cuentas no hay que olvidar que estos cuadros han sido pintados por un hombre, ni que, en este matadero, el hombre es siempre la víctima, la presa que se abate. Legítimamente o no, estas mujeres son siempre mantenidas, siempre es el hombre quien paga, en todos los sentidos de la palabra: domesticado o asesinado.

Y para finalizar nuestro análisis, una rápida referencia a la presencia de unos pocos retratos que nos interesan de manera especial por su intertexto pictórico que permite instaurar una interacción entre el texto periodístico literario y el cuadro. Insinuándose entre la masa de parisinas que vampirizan al hombre, aparece una serie de retratos de mujeres anónimas, casi inexistentes, que, por su belleza y atractivo, intentan compensar el carácter nefasto de los anteriores retratos. El mejor ejemplo de cllos sería cl retrato de esa mujer ausente, la viajera que se va sin que el enamorado haya tenido tiempo de 
abordarla ni aun de conocerla; clla es «Celle qui s'en va». Mujer ideal por excelencia, inteligente, encantadora, soñadora y sublime, es la más perfecta representación de la atmósfera decadente cuya evocación se ve reforzada en el texto por la referencia a los pintores favoritos del autor (Burne Jones, Moreau, Puvis de Chavannes, Crane et Whistler), suficiente para traducir la concepción lorrainiana, que es también la de sus contemporáneos, de una belleza etérea. Esta referencia, en relación con uno de los pintores citados, implica dos constituyentes formales: el nombre propio (Burne Jones) y el título de la obra (The Legend fo the Briat Rose) que cumple una función cultural, estética y metafórica ${ }^{18}$.

El nombre propio, Burne Jones aparece como un «designador rígido» que hace referencia no sólo al pintor mismo sino también a uno de los pintores que mayor influencia tuvieron entre los decadentes franceses. El título del cuadro se inscribe también en un código y se incluye en los catálogos. De esta manera, el lector está implicado por la elección del autor, por su subjetividad, y es invitado a la complicidad con el autor teniendo al cuadro como intermediario. A través de esta integración del referente plástico en el tex to escrito, se ofrece al lector la posibilidad de compartir el sistema de interpretación y de visión del autor así como de traducir las correspondencias que existen entre retrato literario y retrato pictórico. En el caso que nos ocupa, esta referencia se ve reforzada por la presencia de un metalenguaje pictórico que pretende describir el cuadro del que se trata.

«Celle qui s'en va» responde a la estética simbolista, creando una imagen sensual muy sugerente y capaz de despertar los sentidos y de hacer soñar con un mundo mágico, alejado del mundo terrestre; el lenguaje empleado por Lorrain parece ajustarse a este ideal:

«Sur les ciels verdissants d'automne, ces ciels de turquoise malade, striés de jaune et de pourpre, qui sentent déjà l'hiver, sa fine silhouette de voyageuse évoque des regrets d'intéricurs, de tendres exils à deux dans des climats plus chauds (...)» (p. 109).

Puede establecerse una analogía entre esta mujer ausente e inaccesible cuyo perfil «cubierto de tul gris» aparece «lejano e irreal», y las figuras ambigüas de los pintores simbolistas a los que hemos hecho referencia anteriormente.

Literatura y pintura se dan cita para entretejer toda una red de influencias e interrelaciones.

« ¿Cerdos, habéis hecho de mí un periodista!», exclamaba Lorrain deplorando no poder consagrarse por entero a la «literatura», entendiendo por ésta la actividad narrativa, teatral o bien aquella otra pasión, más juvenil, por los versos parnasianos. Sin embargo hoy parece evidente, como lo anunciábamos al principio de nuestro trabajo, que su «destino» literario se inscribía más bien en esta línea y que su «genio» se manifiesta más claramente en la utilización de una prosa inmediata, provocada por el instante y que se

ix Cfr. B. Votmitoux, art cil. (1985), pp. 198-199. 
expresa en una escritura rápida, intuitiva y visual. Es, pues, al periodista al que admiramos en Lorrain, al observador atento que ha sabido pintar perfectamente a la parisina de fin de siglo. Actrices, literatas, burguesas y prostitutas pueblan el París de Lorrain y, en esta galería de retratos que nos presenta, aparece una dominante prosopográfica en la descripción de las actrices y de las prostitutas, mientras que la etopeya prevalece en las literatas y burguesas, figuras que provocan terror y no inspiran nunca confianza a Lorrain. El mismo atraído por el espectáculo del hampa y los márgenes dudosos, se muestra más complaciente con las mujeres que viven al margen de una sociedad instalada y bien pensante y cuyo ejercicio de la voluptuosidad parece más cerca de las inclinaciones de Lorrain, ya que, como él mismo decía: «Les raisonnables auront duré, les voluptueux auront vécu» (4).

Por lo que se refiere a la composición de estos cuadros, la técnica empleada por Lorrain es esencialmente la misma: ya sea el juicio crítico contra las mujeres razonables o la mirada benévola que otorga a las voluptuosas, al final retoma el principio del texto y, por esta circularidad, a la ful gurancia del trazo inicial del que hablaba Morand responde - a modo de rúbrica- la instantánea de una imagen final que, relente o perfume, querría transmitir al lector la verdad del retrato. 